九州大学学術情報リポジトリ

Kyushu University Institutional Repository

\title{
Intrasubgeneric and Interploid Cross
} Compatibility in Evergreen and Deciduous Azaleas

\section{Sakai, Kaori}

Faculty of Agriculture, Kyushu Unviersity

Ozaki, Yukio

Faculty of Agriculture, Kyushu Unviersity

Hiramatsu, Michikazu

Faculty of Agriculture, Kyushu Unviersity

Wakana, Akira

Faculty of Agriculture, Kyushu Unviersity

他

https://doi.org/10.5109/4713

出版情報：九州大学大学院農学研究院紀要. 51 (1)，pp.73-81，2006-02-01. Faculty of Agriculture， Kyushu University

バージョン:

権利関係: 


\title{
Intrasubgeneric and Interploid Cross Compatibility in Evergreen and Deciduous Azaleas
}

\author{
Kaori SAKAI*, Yukio OZAKI, Michikazu HIRAMATSU', \\ Akira WAKANA ${ }^{1}$ and Hiroshi OKUBO ${ }^{1}$
}

\author{
Laboratory of Agricultural Ecology, Division of Agricultural Ecology, Department of Plant Resources, \\ Faculty of Agriculture, Kyushu University, Fukuoka 811-2307, Japan \\ (Received October 28, 2005 and accepted November 16, 2005)
}

\begin{abstract}
Twenty-three diploids, six triploids, nine tetraploids and four mixoploids in evergreen azaleas and eight diploids and five tetraploids in deciduous azaleas were identified by flow cytometric analysis of ploidy in species, interspecific hybrids and cultivars. Sizes of guard cells and pollen grains tended to increase in proportion to the increase in the ploidy. Intrasubgeneric and intra- or interploid hybridizations were conducted with evergreen and deciduous azaleas. Tetraploids of evergreen azaleas had relatively high crossability as seed and/or pollen parents, and triploid evergreen azaleas had fertility as seed parents only. Deciduous tetraploids had high pollen fertility and, therefore, capsules set relatively frequently in the interploid crosses of $2 \mathrm{x} \times 4 \mathrm{x}$. No capsules set in the reciprocal crosses.
\end{abstract}

\section{INTRODUCTION}

The fundamental chromosome number in Rhododendron is 13 (Sax, 1930). The species of the lepidote group of the genus are highly polyploid, whereas those of the elepidote group such as subgenera of Tsutsusi and Pentanthera, which are commonly called as evergreen and deciduous azaleas respectively, are mostly diploid (Janaki Ammal, 1950). All reported species of evergreen azaleas are diploid $(2 n=2 x=26)$. There are a few cultivars of triploid and tetraploid in Satsuki Hybrid (Hosoda et al., 1953), and triploid, tetraploid and mixoploid in Belgian Hybrid (Pryor and Frazier, 1970; Heursel and De Roo, 1981; De Schepper et al., 2001a). Deciduous azalea species contains two tetraploid species; they are $R$. calendulaceum and $R$. canadense, and the both are native of the eastern North America (Sax, 1930). Kehr (1987) pointed out that some cultivars developed from subgenus Pentanthera might contain some polyploid cultivars, but the fact has not been shown.

Hybridization within or between species has certainly played an important role in ornamental plant breeding (Horn, 2003; Van Tuyl and Lim, 2003). Intraor interspecific cross compatibilities among evergreen and deciduous azaleas were investigated precisely (Noguchi, 1932; Akabane et al., 1971; Yamaguchi and Hirata, 1986; Yamaguchi et al., 1985). While the crosses between distantly related species belonging to different subgenera in Rhododendron were difficult or impossible, high cross compatibilities between closely related species within the same section were clarified with some exceptions. Thus, intra- or interspecific cross compatibilities in azaleas have been the objectives of studies for long time, but little is known about intra- or interspe-

${ }^{1}$ Laboratory of Horticultural Science, Division of Agricultural Botany, Department of Plant Resources, Faculty of Agriculture, Kyushu University, Fukuoka 812-8581, Japan

* Corresponding author (E-mail: sakai@farm.kyushu-u.ac.jp) cific cross compatibilities with respect to ploidy levels. The deficient knowledge seems to be caused by the little information about polyploids in azaleas.

In this study, ploidy levels of evergreen and deciduous azalea species, interspecific hybrids and cultivars were assessed by flow cytometric analysis. Flow cytometric analysis has been introduced to determine ploidy level in Rhododendron as the alternative to chromosome counting (Vainola, 2000; De Schepper et al., 2001a, b). The relation among the ploidy levels of the plants and sizes of their guard cells and pollen grains were also investigated to ascertain the ploidy levels determined by flow cytometry. Intrasubgeneric and interploid hybridizations in evergreen and deciduous azaleas were performed and their interploid cross compatibilities were determined.

\section{MATERIALS AND METHODS}

\section{Plant materials}

Plant materials used in this study are listed in Table 1. Ploidy levels of 13 plants of three species (R. eriocarpum, $R$. indicum and $R$. kiusianum), five plants of interspecific hybrids $(R$. kiusianum $\times R$. eriocarpum) and 24 cultivars of evergreen azaleas were investigated. All of the species, interspecific hybrids and cultivars of evergreen azaleas used in this study belong to subgenus Tsutsusi. Ploidy levels were also determined in eight plants of $R$. japonicum, six plants of $R$. japonicum f. flavum and five cultivars of Knap Hill Hybrid and Mollis Hybrid. They all belong to subgenus Pentanthera, so-called deciduous azaleas. Among them, (R. kiusianum $\times R$. eriocarpum) No. 5 and $R$. japonicum $\mathrm{f}$. flavum No. 6 were raised from the buds treated with colchicine (chromosome doubling was expected).

\section{Assessment of ploidy levels with flow cytometry}

The assessment of ploidy levels was conducted by flow cytometry. Fully expanded young leaves or young roots were chopped with a sharp razor blade in nuclei 
Table 1. Species, interspecific hybrids and cultivars of evergreen and deciduous azaleas used for ploidy determination by flow cytometry.

\begin{tabular}{|c|c|}
\hline Plant & Description \\
\hline $\begin{array}{l}\text { Evergreen azaleas } \\
\quad \text { R. eriocarpum (No. } 1,2,3)^{z} \\
\quad \text { R. indicum (No. } 1,2,3,4,5,6,7) \\
\quad \text { R. kiusianum (No. } 1,2,3 \text { ) }\end{array}$ & Subg. Tsutsusi. \\
\hline $\begin{array}{r}R . \text { kiusianum } \times R \text { eriocarpum } \\
\left(\text { No. } 1,2,3,4,5^{\mathrm{y}}\right)\end{array}$ & $\begin{array}{l}\text { Interspecific hybrid, subg. Tsutsusi } \times \\
\text { subg. Tsutsusi. }\end{array}$ \\
\hline $\begin{array}{l}\text { 'Ayaka', 'Eiganohomare', 'Eiko', 'Goka } \\
\text { 'Gyoko', 'Hakatajiro', 'Hoshuku', 'Hos' } \\
\text { 'Isshonoharu', 'Kobai', 'Koyo', } \\
\text { 'Mibunohana', 'Miharu', 'Meicho', } \\
\text { 'Sachinoharu', 'Shinsen', 'Suisen', } \\
\text { 'Shunka', 'Taihei', 'Taikonotsuki', } \\
\text { 'Daisetsuzan', 'Takarabune', 'Yuhime' }\end{array}$ & $\begin{array}{l}\text { Satsuki Hybrid, an intrasubgeneric } \\
\text { hybrid group in subg. Tsutsusi. }\end{array}$ \\
\hline 'Horiuchikanzaki' & $\begin{array}{l}\text { Inter-group hybrid between Belgian } \\
\text { Hybrid and Hirado Hybrid in subg. } \\
\text { Tsutsusi. }\end{array}$ \\
\hline $\begin{array}{l}\text { Deciduous azaleas } \\
R . \text { japonicum }(\text { No. } 1,2) \\
R . \text { japonicum f. flavum } \\
\left.\text { (No. } 1,2,3,4,5,6^{y}\right)\end{array}$ & Subg. Pentanthera. \\
\hline $\begin{array}{l}\text { 'Golden Flare', 'Golden Sunset', } \\
\text { 'Klondyke', 'Melford Yellow' }\end{array}$ & $\begin{array}{l}\text { Knap Hill Hybrid, an intrasubgeneric } \\
\text { hybrid group in subg. Pentanthera. }\end{array}$ \\
\hline 'Directeur Moerlands' & $\begin{array}{l}\text { Mollis Hybrid, an intrasubgeneric hybrid } \\
\text { group in subg. Pentanthera. }\end{array}$ \\
\hline
\end{tabular}

${ }^{2}()=$ individual plant number.

${ }^{y}$ Seedings of $(R$. kiusianum $\times R$. eriocarpum $)$ No. 5 and $R$. japonicum f. flavum No. 6 were derived from buds treated with colchicine.

extraction buffer (High resolution DNA kit, Partec), and the suspension containing released nuclei was passed through a $50 \mu \mathrm{m}$ nylon mesh filter. Then the nuclei in filtrate were stained with four times volumes of staining solution (High resolution DNA kit, Partec) containing 4'-6-diamidino-2-phenylindole (DAPI). After shaking the solution gently, samples were analyzed with a flow cytometer (PA Ploidy Analyzer, Partec). Relative DNA content was estimated according to the prominent peaks in each measurement.

\section{Measurement of pollen grain sizes}

Pollen grains were collected at the full bloom stage in each plant, and observed with an optical microscope after staining with aceto-carmine for 10 minutes. The pollen grains were classified into perfect pollen with densely stained cytoplasm or empty pollen that was not stained. The diameters of twenty perfect pollen grains in three replications were measured.

\section{Measurement of guard cell sizes}

Impressions of guard cells were made by painting the abaxial surface of three mature leaves each from diploid, triploid, tetraploid and mixoploid plants with clear fingernail polish. After the impressions had been dried, they were stripped off by a transparent adhesive tape, sealed on slide glasses, and used to measure the guard cell length under a optical microscope.

\section{Pollen fertility}

Pollen fertility of evergreen and deciduous azaleas was examined. Pollen grains were collected at their full bloom stage, and observed with an optical microscope after staining with aceto-carmine for 10 minutes. Pollen fertility was evaluated by number of perfect pollen grains (densely stained pollen grains) divided by the number of pollen grains observed. More than 400 pollen grains in three replications were observed for estimation of fertility in each accession.

\section{Intra- and interploid crosses}

Intrasubgeneric intra- and interploid crosses among evergreen and deciduous azaleas were conducted from 19 April to 9 June 2002 or from 21 April to 24 June 2003. Flowers of pistillate parents were emasculated and covered with paper bags about seven days before anthesis and pollinated at anthesis with fresh or one-year-old pollen stored at $-20^{\circ} \mathrm{C}$. Viability of azalea pollen after the storage for years at $-20^{\circ} \mathrm{C}$ has been confirmed (Rouse, 1984; Kehr, 1987). All pollinated flowers were re-covered with paper bags to prevent them from contamination by undesirable pollen. Thirteen diploids, five triploids, four tetraploids and three mixoploids in evergreen azaleas, and six diploids and five tetraploids in deciduous azaleas were chosen as cross parents. A total of 219 pollinations in 52 cross combinations among evergreen azaleas and 202 pollinations in 28 crosses among deciduous azaleas were made. Capsule setting was 
observed 90 days after crossings. Capsules were collected 180 days after pollination, and number of seeds per capsule and the percentages of perfect seeds were scored.

When mixoploids produce $2 \mathrm{x}$-gamate judging from the results of flow cytometric analysis and measurements of pollen grain sizes, the results obtained from crosses with the mixoploids were included in those from crosses with tetraploids in Table 6.

\section{RESULTS}

\section{Assessment of ploidy levels}

Typical histograms of flow cytometric analysis in diploid, triploid, tetraploid and mixoploid $(2 \mathrm{x}+4 \mathrm{x})$ plants are shown in Fig. 1. Each ploidy level was clearly identified by the analysis using young leaves. The fluorescent intensities at prominent peaks in diploid, triploid and tetraploid plants were about 100, 150 and 200, respectively. The plants showing two peaks of fluorescent intensities at 100 and 200 were judged to be

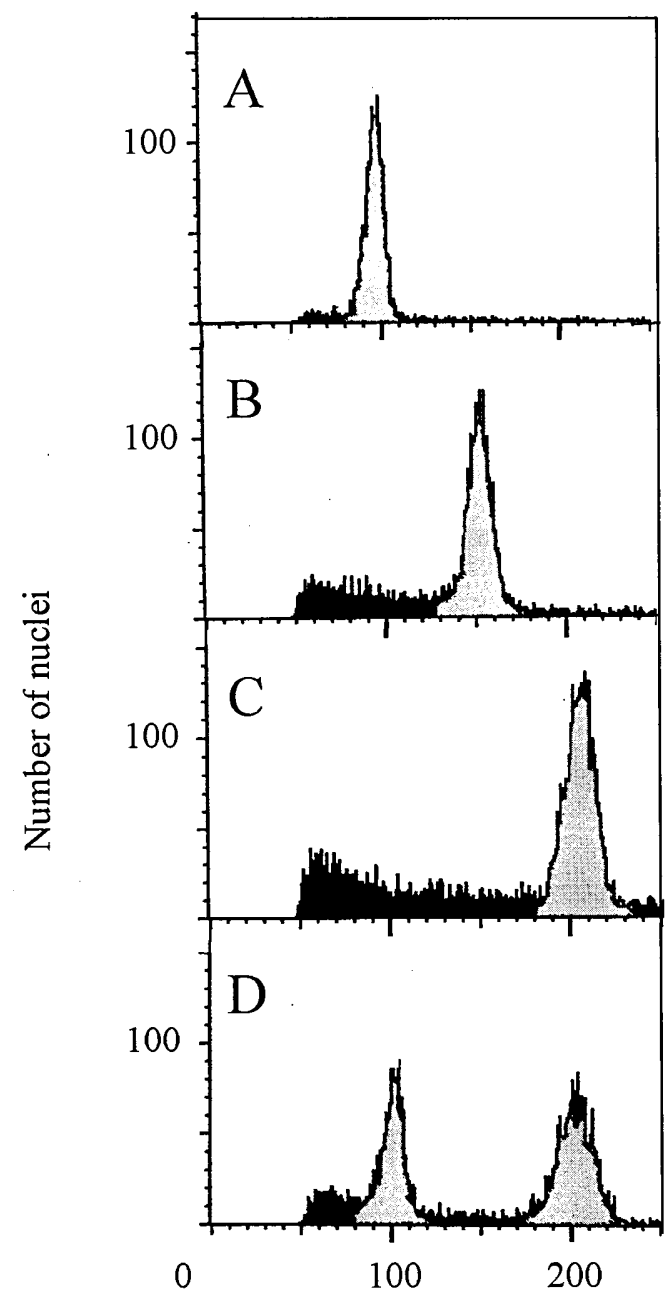

Fluorescent intensity

Fig. 1. Flow cytometric histogram patterns of diploid, triploid, tetraploid and mixoploid. A: diploid evergreen azalea $R$. indicum No. 1, B: triploid evergreen azalea 'Daisetsuzan', C: tetraploid azalea 'Hoshun' and D: mixoploid azalea 'Miharu'. mixoploids $(2 x+4 x)$. Similar results were obtained from the roots in diploids, triploids and tetraploids by the flow cytometric analysis. Although 'Miharu' and 'Shinsen' were identified to be mixoploids with their leaves, their roots had only tetraploid nuclei. Twenty-three diploid, six triploid, nine tetraploid and four mixoploid evergreen azaleas, and eight diploid and five tetraploid deciduous azaleas were identified by flow cytometry (Table 2).

\section{Pollen grain sizes}

Most of the pollen grains from the diploid azaleas were well stained and plump, while empty pollen grains were found in triploid, tetraploid and mixoploid azaleas in relatively high frequencies (Fig. 2). The sizes of well-stained pollen grains tended to increase in proportion to the increase in ploidy levels (Table 3). Stained pollen grains from the mixoploid evergreen azaleas were as large as those from the tetraploid evergreen azalea.

\section{Guard cell sizes}

Stomatal guard cells of the tetraploid 'Hoshun' were longer than those of the diploids (Fig. 3, Table 4). Although the guard cells of mixoploid 'Shinsen', a Satsuki Hybrid, were smaller than those of the diploids, 'Miharu' had much larger guard cells than diploid plants.

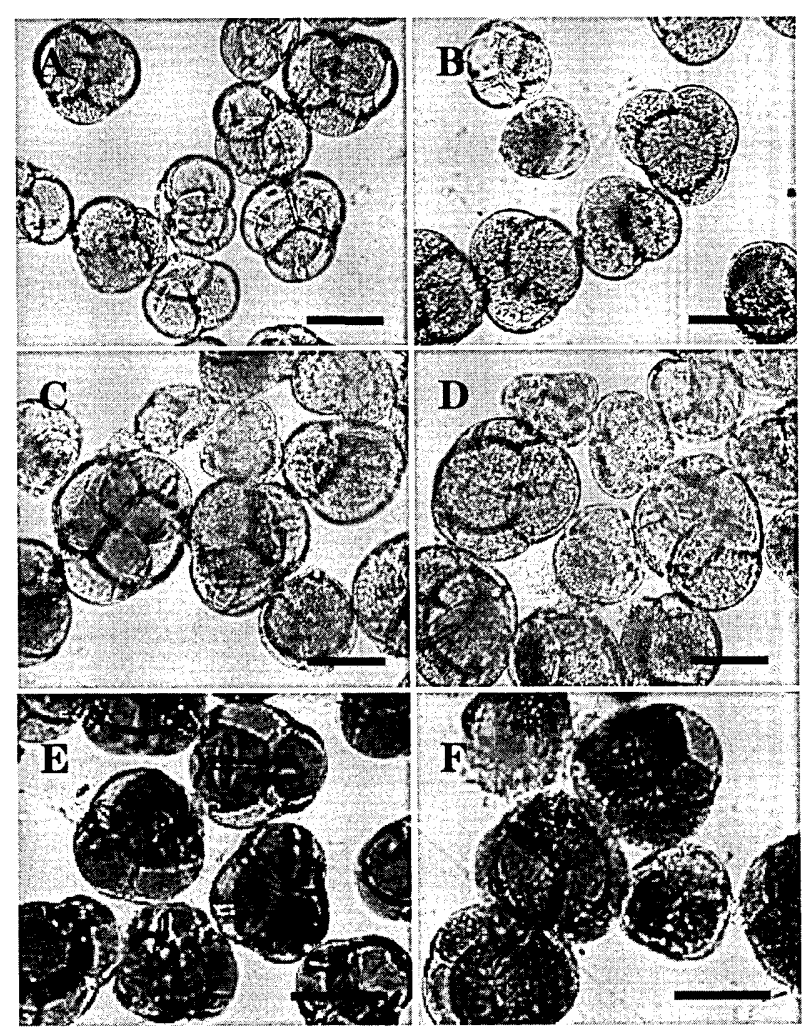

Fig. 2. Pollen grains of diploid, triploid, tetraploid and mixoploid plants.

A: diploid evergreen azalea 'Mibunohana', B: triploid evergreen azalea 'Goka', C: tetraploid evergreen azalea 'Hoshun', D: mixoploid evergreen azalea 'Miharu', E: diploid deciduous azalea $R$. japonicum f. flavum (No. 1) and F: tetraploid deciduous azalea 'Klondyke'. Scale $=50 \mu \mathrm{m}$. 
Table 2. Ploidy levels of evergreen and deciduous azaleas determined by flow cytometry.

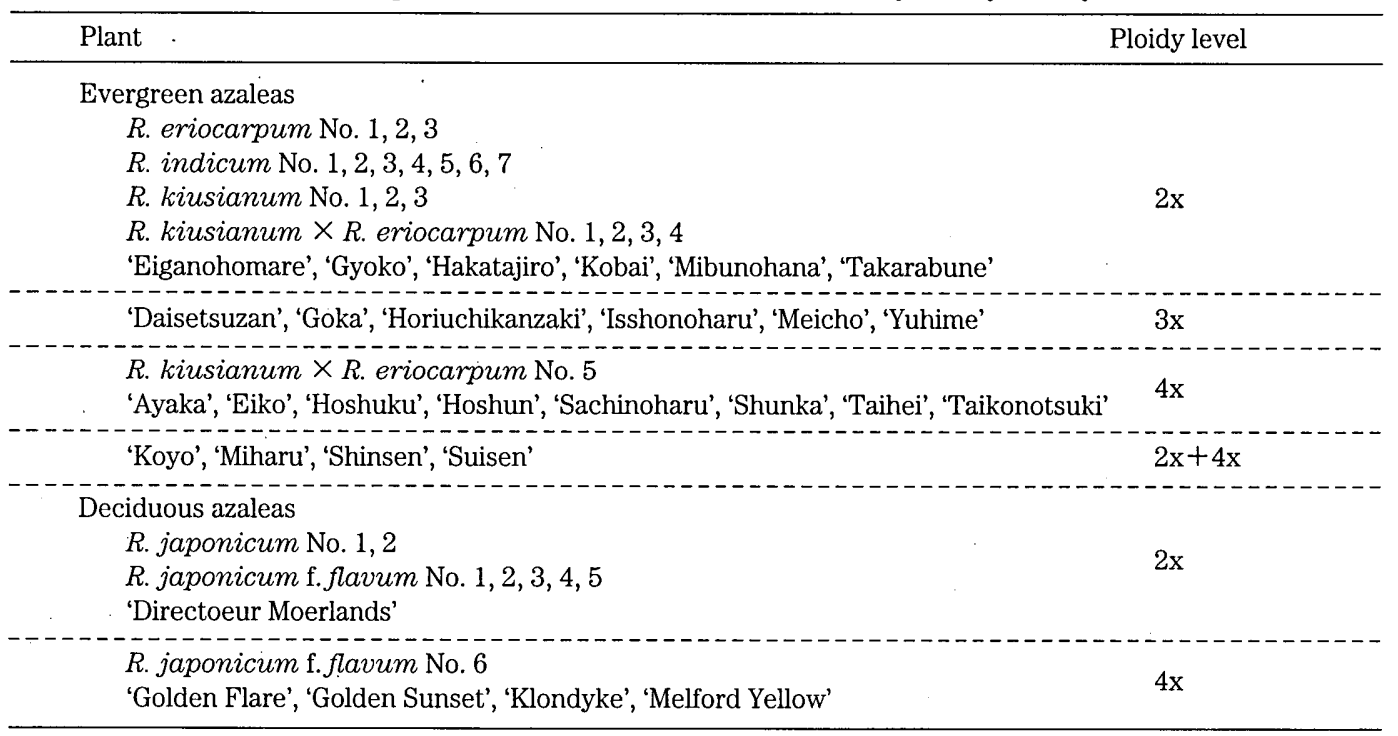

Table 3. Mean diameter of pollen grains well-stained with acetocarmine in various ploids of evergreen and deciduous azaleas.

\begin{tabular}{|c|c|c|}
\hline Plant & Ploidy level & $\begin{array}{c}\text { Mean } \\
\text { diameter of } \\
\text { pollen grains } \\
(\mu \mathrm{m}) \\
\end{array}$ \\
\hline \multicolumn{3}{|l|}{ Evergreen azaleas } \\
\hline R. indicum No. 3 & $2 \mathrm{x}$ & 39.1 \\
\hline R. indicum No. 5 & $2 \mathrm{x}$ & 35.0 \\
\hline 'Mibunohana' & $2 \mathrm{x}$ & 40.7 \\
\hline 'Daisetsuzan' & $3 x$ & 42.2 \\
\hline 'Goka' & $3 \mathrm{x}$ & 44.6 \\
\hline 'Hoshun' & $4 \mathrm{x}$ & 48.7 \\
\hline 'Miharu' & $2 x+4 x$ & 47.9 \\
\hline 'Shinsen' & $2 x+4 x$ & 50.0 \\
\hline \multicolumn{3}{|l|}{ Deciduous azaleas } \\
\hline R. japonicum f. flavum No. 1 & $2 \mathrm{x}$ & 41.3 \\
\hline 'Klondyke' & $4 \mathrm{x}$ & 44.8 \\
\hline
\end{tabular}

Table 4. Sizes of guard cells in various ploids of evergreen and deciduous azaleas.

\begin{tabular}{lll}
\hline Plant & Ploidy level & $\begin{array}{l}\text { Mean diameter } \\
\text { of guard } \\
\text { cells }(\mu \mathrm{m})\end{array}$ \\
\hline Evergreen azaleas & & \\
$\quad$ R. indicum No. 3 & $2 \mathrm{x}$ & 19.2 \\
$\quad$ R. indicum No. 5 & $2 \mathrm{x}$ & 19.3 \\
'Mibunohana' & $2 \mathrm{x}$ & 18.2 \\
'Daisetsuzan' & $3 \mathrm{x}$ & 16.1 \\
'Goka' & $3 \mathrm{x}$ & 19.8 \\
'Hoshun' & $4 \mathrm{x}$ & 23.9 \\
'Miharu' & $2 \mathrm{x}+4 \mathrm{x}$ & 21.3 \\
'Shinsen' & $2 \mathrm{x}+4 \mathrm{x}$ & 15.8 \\
\hline Deciduous azaleas & & \\
R. japonicum $\mathrm{f}$. flavum No. 1 & $2 \mathrm{x}$ & 10.2 \\
'Klondyke' & $4 \mathrm{x}$ & 16.3 \\
\hline
\end{tabular}
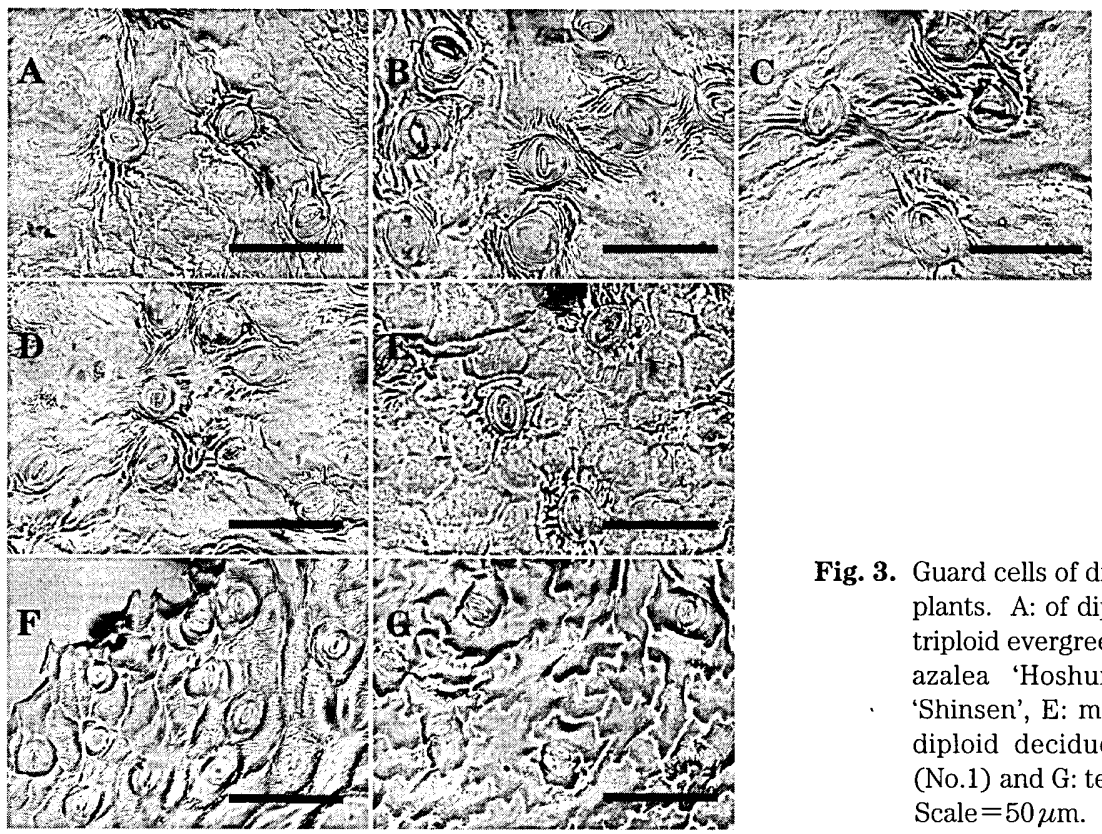

Fig. 3. Guard cells of diploid, triploid, tetraploid and mixoploid plants. A: of diploid evergreen azalea 'Mibunohana', B: triploid evergreen azalea 'Goka', C: tetraploid evergreen azalea 'Hoshun', D: mixoploid evergreen azalea 'Shinsen', E: mixoploid evergreen azalea 'Miharu', F: diploid deciduous azalea $R$. japonicum f. flavum (No.1) and G: tetraploid deciduous azalea 'Klondyke'. Scale $=50 \mu \mathrm{m}$. 
Table 5. Pollen fertility in evergreen and deciduous azaleas with various ploidy.

\begin{tabular}{|c|c|c|}
\hline Plant & Ploidy level & Pollen fertility (\%) \\
\hline \multicolumn{3}{|l|}{ Evergreen azaleas } \\
\hline R. indicum No. 3 & $2 \mathrm{x}$ & 88.8 \\
\hline R. indicum No. 5 & $2 \mathrm{x}$ & 99.3 \\
\hline 'Mibunohana' & $2 \mathrm{x}$ & 84.0 \\
\hline 'Daisetsuzan' & $3 \mathrm{x}$ & 72.0 \\
\hline 'Goka’’ & $3 \mathrm{x}$ & 66.6 \\
\hline 'Hoshun' & $4 \mathrm{x}$ & 60.5 \\
\hline 'Miharu' & $2 x+4 x$ & 23.4 \\
\hline 'Shinsen' & $2 x+4 x$ & 90.6 \\
\hline \multicolumn{3}{|l|}{ Deciduous azaleas } \\
\hline R. japonicum f. flavum No. 1 & $2 \mathrm{x}$ & 95.9 \\
\hline 'Klondyke' & $4 \mathrm{x}$ & 89.1 \\
\hline
\end{tabular}

\section{Pollen fertility}

Pollen fertility of diploid evergreen azaleas was higher (84.0-99.3\%) than that of triploids (66.6 and $72.0 \%)$ and tetraploid (60.5\%) evergreen azaleas, while in two mixoploid evergreen azaleas it was different (23.4 and 90.6\%) (Table 5). Both diploid and tetraploid deciduous azaleas had relatively high pollen fertility; 95.9 and $89.1 \%$, respectively.

\section{Intra- and interploid crosses}

In intraploid crosses with evergreen azaleas, $2 \mathrm{x} \times$ $2 \mathrm{x}$ crosses brought higher capsule set $(95.2 \%)$ than $4 \mathrm{x}$ $\times 4 \mathrm{x}$ crosses (48.7\%) (Table 6). No capsules set in any of the crosses with triploids when used as pollen parents. The capsule set was the highest in $3 \mathrm{x} \times 2 \mathrm{x}$ (72.2\%) among interploid crosses with evergreen azaleas (Table 6).
Capsules from $2 \mathrm{x} \times 2 \mathrm{x}$ crosses were also obtained with higher rate $(87.3 \%)$ than those from $4 \mathrm{x} \times 4 \mathrm{x}$ (6.8\%) among the intraploid crosses with deciduous azaleas, (Table 7). Although capsules were obtained from $2 \mathrm{x} \times 4 \mathrm{x}$ crosses, no capsules set in the reciprocal crosses, $4 \mathrm{x} \times 2 \mathrm{x}$ (Table 7).

More than 500 seeds per capsule were obtained from the $2 \mathrm{x} \times 2 \mathrm{x}$ crosses with evergreen azaleas and relatively many seeds (more than 300 per capsule) were produced in the crosses of $4 \mathrm{x} \times 4 \mathrm{x}$ on an average. Most of them were perfect seeds ( 97.6 for the $2 \mathrm{x} \times 2 \mathrm{x}$ and $93.2 \%$ for the $4 \mathrm{x} \times 4 \mathrm{x}$ ) (Table 6). Average number of seeds per capsule obtained from the interploid crosses ranged from 62.1 for the $3 \mathrm{x} \times 4 \mathrm{x}$ to 268.2 for the $3 \mathrm{x} \times$ $2 \mathrm{x}$. Perfect seeds were obtained relatively frequently (more than $80 \%$ ) from the interploid crosses, on an average, except for the $3 \mathrm{x} \times 4 \mathrm{x}$ crosses $(72.2 \%)$. In the

Table 6. Capsule set rates, number of seeds and percentages of perfect seeds in intra- and interploid crosses with evergreen azaleas.

\begin{tabular}{|c|c|c|c|c|c|c|}
\hline Seed parent & Pollen parent & $\begin{array}{l}\text { No. of pollinated } \\
\text { flowers }\end{array}$ & $\begin{array}{l}\text { No. of } \\
\text { capsules }\end{array}$ & $\begin{array}{l}\% \text { of capsules } \\
\text { set }\end{array}$ & $\begin{array}{l}\text { No. of seeds } \\
\text { per capsule }\end{array}$ & $\begin{array}{l}\% \text { of perfect } \\
\text { seeds }\end{array}$ \\
\hline $\begin{array}{l}\text { Intra-ploid crosses } \\
{[2 x]}\end{array}$ & \multicolumn{6}{|l|}{$[\mathbf{2 x}]$} \\
\hline R. eriocarpum No. 1 & R. eriocarpum No. 2 & 6 & 6 & 100 & 903.8 & 99.2 \\
\hline R. eriocarpum No. 2 & R. indicum No. 1 & 8 & 8 & 100 & 609.1 & 98.1 \\
\hline R. eriocarpum No. 2 & 'Eiganohomare' & 2 & 2 & 100 & 66.0 & 95.5 \\
\hline R. eriocarpum No. 2 & 'Hakatajiro' & 5 & 5 & 100 & 332.4 & 96.5 \\
\hline R. indicum No. 1 & R. eriocarpum No. 2 & 5 & 5 & 100 & 652.8 & 97.0 \\
\hline R. indicum No. 2 & R. eriocarpum No. 2 & 5 & 4 & 80 & 800.5 & 97.0 \\
\hline R. indicum No. 3 & R. eriocarpum No. 2 & 3 & 3 & 100 & 392.3 & 99.2 \\
\hline R. indicum No. 4 & R. indicum No. 1 & 3 & 3 & 100 & 654.0 & 98.0 \\
\hline 'Mibunohana' & R. indicum No. 3 & 5 & 4 & 80 & 449.5 & 94.3 \\
\hline \multicolumn{2}{|l|}{$2 \mathrm{x} \times 2 \mathrm{x}$ Subtotal } & 42 & 40 & 95.2 & 587.3 & 97.6 \\
\hline$[3 \mathbf{x}]$ & \multicolumn{6}{|l|}{$[\mathbf{3 x}]$} \\
\hline 'Goka' & 'Isshonoharu' & 2 & 0 & 0 & - & - \\
\hline 'Horiuchikanzaki' & 'Goka' & 4 & 0 & 0 & $=$ & $=$ \\
\hline \multicolumn{2}{|l|}{$3 x \times 3 x$ Subtotal } & 6 & 0 & 0 & - & - \\
\hline$[4 x]$ & {$[2 x+4 x]$} & & & & & \\
\hline 'Hoshuku' & 'Shinsen’ & 6 & 1 & 16.6 & 138.0 & 93.5 \\
\hline 'Hoshun' & 'Miharu' & 5 & 0 & 0 & - & - \\
\hline 'Hoshun' & 'Shinsen' & 5 & 4 & 80 & 410.7 & 85.5 \\
\hline
\end{tabular}


Table 6. Continued

\begin{tabular}{|c|c|c|c|c|c|c|}
\hline Seed parent & Pollen parent & $\begin{array}{l}\text { No. of pollinated } \\
\text { flowers }\end{array}$ & $\begin{array}{l}\text { No. of } \\
\text { capsules }\end{array}$ & $\begin{array}{l}\% \text { of capsules } \\
\text { set }\end{array}$ & $\begin{array}{l}\text { No. of seeds } \\
\text { per capsule }\end{array}$ & $\begin{array}{l}\% \text { of perfect } \\
\text { seeds }\end{array}$ \\
\hline $\begin{array}{l}\text { 'Sachinoharu' } \\
{[\mathbf{2 x}+\mathbf{4 x}]}\end{array}$ & $\begin{array}{l}\text { ‘Suisen' } \\
{[\mathbf{4 x}]}\end{array}$ & 2 & 0 & 0 & - & - \\
\hline 'Miharu' & 'Hoshun' & 5 & 4 & 80 & 310.3 & 97.2 \\
\hline 'Miharu' & 'Sachinoharu' & 5 & 3 & 60 & 194.3 & 97.3 \\
\hline 'Shinsen' & 'Hoshun' & 5 & 4 & 80 & 495.0 & 95.5 \\
\hline 'Shinsen' & 'Sachinoharu' & 6 & 3 & 50 & 289.3 & 94.1 \\
\hline $4 x \times 4 x$ Subtotal & & 39 & 19 & 48.7 & 339.6 & 93.2 \\
\hline \multicolumn{6}{|l|}{ Inter-ploid crosses } & \\
\hline R. eriocarpum No. 2 & 'Goka' & 9 & 0 & 0 & - & - \\
\hline R. eriocarpum No. 2 & 'Daisetsuzan' & 5 & 0 & 0 & - & - \\
\hline R. eriocarpum No. 2 & 'Horiuchikanzaki' & 5 & 0 & 0 & - & - \\
\hline R. indicum No. 6 & 'Daisetsuzan' & 5 & 0 & 0 & - & - \\
\hline R. indicum No. 1 & 'Horiuchikanzaki' & 5 & 0 & 0 & - & - \\
\hline 'Mibunohana' & 'Daisetsuzan' & 2 & 0 & 0 & - & - \\
\hline \multicolumn{2}{|l|}{$2 \mathrm{x} \times 3 \times$ Subtotal } & 31 & 0 & 0 & - & - \\
\hline$[2 \mathrm{x}]$ & {$[4 x]$} & & & & & \\
\hline R. eriocarpum No. 1 & 'Taikonotsuki' & 2 & 2 & 100 & 35.0 & 100 \\
\hline R. eriocarpum No. 3 & 'Ayaka' & 4 & 0 & 0 & - & - \\
\hline 'Mibunohana' & 'Hoshun' & 5 & 5 & 100 & 136.0 & 84.7 \\
\hline$[2 \mathrm{x}]$ & {$[2 x+4 x]$} & & & & & \\
\hline R. eriocarpum No. 2 & 'Shinsen' & 5 & 5 & 100 & 456.6 & 95.7 \\
\hline R. eriocarpum No. 2 & 'Suisen' & 5 & 1 & 20 & 35.0 & 88.6 \\
\hline R. indicum No. 7 & 'Shinsen' & 3 & 1 & 33.3 & 48.0 & 89.6 \\
\hline R. indicum No. 7 & 'Miharu' & 5 & 5 & 100 & 35.8 & 98.3 \\
\hline \multicolumn{2}{|l|}{$2 x \times 4 x$ Subtotal } & 29 & 19 & 65.5 & 173.4 & 93.5 \\
\hline$[3 \mathbf{x}]$ & {$[2 \mathrm{x}]$} & & & & & \\
\hline 'Daisetsuzan' & R. indicum No. 3 & 1 & 1 & 100 & 201.0 & 74.1 \\
\hline 'Daisetsuzan' & R. indicum No. 5 & 1 & 0 & 0 & - & - \\
\hline 'Daisetsuzan' & R. indicum No. 6 & 5 & 3 & 60 & 201.3 & 92.7 \\
\hline 'Daisetsuzan' & R. eriocarpum No. 2 & 4 & 3 & 75 & 239.3 & 91.5 \\
\hline 'Goka' & R. indicum No. 3 & 2 & 2 & 100 & 145.0 & 70.7 \\
\hline 'Horiuchikanzaki' & R. indicum No. 3 & 5 & 4 & 80 & 418.3 & 86.2 \\
\hline \multicolumn{2}{|l|}{$3 \times \times 2 x$ Subtotal } & 18 & 13 & 72.2 & 268.2 & 86.4 \\
\hline$[3 \mathbf{x}]$ & {$[4 x]$} & & & & & \\
\hline 'Goka' & 'Hoshun' & 2 & 2 & 100 & 103.5 & 60.9 \\
\hline$[3 \mathbf{x}]$ & {$[2 x+4 x]$} & & & & & \\
\hline 'Daisetsuzan' & 'Miharu' & 5 & 5 & 100 & 45.5 & 82.4 \\
\hline 'Horiuchikanzaki' & 'Miharu' & 5 & 0 & 0 & - & - \\
\hline \multicolumn{2}{|l|}{$3 x \times 4 x$ Subtotal } & 12 & 7 & 58.3 & 62.1 & 72.2 \\
\hline$[4 x]$ & {$[2 \mathrm{x}]$} & & & & & \\
\hline 'Hoshuku' & R. indicum No. 6 & 5 & 0 & 0 & - & - \\
\hline $\begin{array}{l}\text { 'Hoshun' } \\
{[\mathbf{2 x}+\mathbf{4} \mathbf{x}]}\end{array}$ & R. indicum No. 5 & 5 & 1 & 20 & 65.0 & 73.8 \\
\hline 'Miharu' & R. indicum No. 5 & 5 & 5 & 100 & 52.0 & 88.5 \\
\hline 'Miharu' & 'Eiganohomare' & 2 & 0 & 0 & - & - \\
\hline 'Shinsen' & R. indicum No. 5 & 5 & 0 & 0 & - & - \\
\hline 'Shinsen' & R. indicum No. 6 & 5 & 2 & 40 & 115.0 & 81.7 \\
\hline 'Shinsen' & R. eriocarpum No. 2 & 2 & 0 & 0 & - & - \\
\hline \multicolumn{2}{|l|}{$4 x \times 2 \times$ Subtotal } & 29 & 8 & 27.6 & 69.4 & 84.0 \\
\hline$[4 \mathrm{x}]$ & {$[3 \mathbf{x}]$} & & & & & \\
\hline 'Hoshun' & 'Goka' & 2 & 0 & 0 & - & - \\
\hline $\begin{array}{l}\text { 'Sachinoharu' } \\
{[\mathbf{2 x}+\mathbf{4 x}]}\end{array}$ & $\begin{array}{l}\text { 'Horiuchikanzaki' } \\
{[\mathbf{3 x}]}\end{array}$ & 1 & 0 & 0 & - & - \\
\hline 'Shinsen' & 'Horiuchikanzaki' & 5 & 0 & 0 & - & - \\
\hline 'Shinsen' & 'Daisetsuzan' & 5 & 0 & 0 & - & - \\
\hline \multicolumn{2}{|l|}{$4 \mathrm{x} \times \mathbf{3 x}$ Subtotal } & 13 & 0 & 0 & - & - \\
\hline \multicolumn{2}{|l|}{ Total } & 219 & 106 & 48.4 & 355.8 & 75.0 \\
\hline
\end{tabular}


Table 7. Capsule set rates, number of seeds and percentages of perfect seeds in intra- and interploid crosses with deciduous azaleas.

\begin{tabular}{|c|c|c|c|c|c|c|}
\hline Seed parent & Pollen parent & $\begin{array}{l}\text { No. of pollinated } \\
\text { flowers }\end{array}$ & $\begin{array}{l}\text { No. of } \\
\text { capsules }\end{array}$ & $\begin{array}{l}\% \text { of capsules } \\
\text { set }\end{array}$ & $\begin{array}{l}\text { No. of seeds } \\
\text { per capsule }\end{array}$ & $\begin{array}{l}\% \text { of perfect } \\
\text { seeds }\end{array}$ \\
\hline $\begin{array}{l}\text { Intra-ploid crosses } \\
{[2 \mathrm{x}]}\end{array}$ & \multicolumn{6}{|c|}{ Intra-ploid crosses } \\
\hline R. japonicum f.flavum No. 1 & R. japonicum f. flavum No. 2 & 15 & 11 & 73.3 & 343.7 & 93.4 \\
\hline R. japonicum f. flavum No. 2 & R. japonicum f. flavum No. 1 & 4 & 2 & 50.0 & 192.5 & 84.4 \\
\hline R. japonicum f. flavum No. 1 & R. japonicum No. 2 & 40 & 38 & 95.0 & 379.3 & 94.1 \\
\hline R. japonicum No. 1 & R. japonicum f. flavum No. 1 & 4 & 4 & 100 & 493.7 & 94.6 \\
\hline \multicolumn{2}{|l|}{$2 x \times 2 x$ Subtotal } & 63 & 55 & 87.3 & 373.7 & 93.8 \\
\hline$[4 x]$ & {$[4 \mathbf{x}]$} & & & & & \\
\hline 'Golden Flare' & 'Klondyke' & 6 & 0 & 0 & - & - \\
\hline 'Golden Flare' & 'Golden Sunset' & 6 & 0 & 0 & - & - \\
\hline ‘Golden Sunset' & 'Klondyke' & 5 & 1 & 20.0 & 126.0 & 80.2 \\
\hline 'Golden Sunset' & 'Golden Flare' & 4 & 2 & 50.0 & 235.0 & 90.9 \\
\hline 'Klondyke' & 'Golden Flare' & 5 & 0 & 0 & - & - \\
\hline 'Klondyke' & 'Golden Sunset' & 3 & 0 & 0 & - & - \\
\hline 'Melford Yellow' & R. japonicum f. flavum No. 6 & 5 & 0 & 0 & - & - \\
\hline 'Melford Yellow' & 'Klondyke' & 10 & 0 & 0 & - & - \\
\hline \multicolumn{2}{|l|}{$4 x \times 4 \times$ Subtotal } & 44 & 3 & 6.8 & 198.7 & 88.6 \\
\hline \multicolumn{7}{|l|}{$\begin{array}{l}\text { Inter-ploid crosses } \\
{[2 \mathrm{x}]}\end{array}$} \\
\hline R. japonicum f. flavum No. 1 & R. japonicum f. flavum No. 6 & 10 & 1 & 10.0 & 20.0 & 60.0 \\
\hline R. japonicum f. flavum No. 1 & 'Golden Flare' & 17 & 11 & 64.7 & 131.7 & 78.5 \\
\hline R. japonicum f. flavum No. 1 & 'Klondyke' & 10 & 6 & 60.0 & 192.0 & 85.6 \\
\hline R. japonicum f. flavum No. 1 & 'Melford Yellow' & 7 & 4 & 57.1 & 123.3 & 75.1 \\
\hline R. japonicum f. flavum No. 2 & 'Golden Flare' & 4 & 0 & 0 & - & - \\
\hline R. japonicum f. flavum No. 2 & 'Klondyke' & 5 & 5 & 100 & 75.7 & 59.9 \\
\hline R. japonicum f. flavum No. 4 & 'Golden Sunset' & 5 & 5 & 100 & 413.0 & 62.8 \\
\hline R. japonicum No. 1 & 'Klondyke' & 5 & 5 & 100 & 388.3 & 82.0 \\
\hline \multicolumn{2}{|l|}{$2 x \times 4 x$ Subtotal } & 63 & 37 & 58.7 & 202.7 & 75.0 \\
\hline$[4 x]$ & {$[2 \mathbf{x}]$} & & & & & \\
\hline 'Golden Flare' & R. japonicum f. flavum No. 1 & 4 & 0 & 0 & - & - \\
\hline 'Golden Flare' & R. japonicum f. flavum No. 2 & 4 & 0 & 0 & - & - \\
\hline 'Golden Sunset' & R. japonicum f. flavum No. 1 & 4 & 0 & 0 & - & - \\
\hline 'Golden Sunset' & R. japonicum f. flavum No. 4 & 4 & 0 & 0 & - & - \\
\hline 'Klondyke' & R. japonicum f. flavum No. 1 & 4 & 0 & 0 & - & _- \\
\hline 'Klondyke' & R. japonicum f. flavum No. 2 & 4 & 0 & 0 & - & - \\
\hline 'Klondyke' & R. japonicum No. 2 & 4 & 0 & 0 & - & - \\
\hline 'Melford Yellow' & R. japonicum f. flavum No. 1 & 4 & 0 & 0 & - & - \\
\hline \multicolumn{2}{|l|}{$4 x \times 2 x$ Subtotal } & 32 & 0 & 0 & - & - \\
\hline \multicolumn{2}{|l|}{ Total } & 202 & 95 & 47.0 & 301.6 & 88.8 \\
\hline
\end{tabular}

intraploid crosses with deciduous azaleas, more perfect seeds were obtained from the $2 \mathrm{x} \times 2 \mathrm{x}$ crosses than the $4 \mathrm{x} \times 4 \mathrm{x}$ crosses (Table 7 ). Relatively many perfect seeds were produced in the interploid crosses of $2 \mathrm{x} \times$ $4 \mathrm{x}$.

\section{DISCUSSION}

\section{Polyploids in evergreen and deciduous azaleas}

Numerous studies about ploidy levels in Rhododendron revealed that most evergreen and deciduous azalea species are diploid (Sax, 1930; Janaki Ammal, 1950; Li, 1957; De Schepper et al., 2001a). Ploidy levels of many cultivars were also investigated. Most of them were diploid and a few were polyploids. De Schepper et al. (2001a) determined the ploidy levels of 88 evergreen azalea cultivars by flow cytometry, and found three triploid $(2 n=3 x=39)$ and one mixoploid $(2 n=2 x+4 x)$ cultivars of Belgian Hybrid. Tetraploid sports from diploid cultivars were identified in Belgian Hybrid (De Schepper et al., 2001b). Tetraploid and triploid cultivars of Satsuki Hybrid were reported, i.e., 'Banka', 'Taihei' and 'Wako' were identified to be tetraploid and 'Bangaku' was identified to be triploid by chromosome counting (Hosoda et al., 1953). Most deciduous species are diploids except that $R$. calendulaceum and $R$. canadense are tetraploids (Sax, 1930; Janaki Ammal, 1950; Li, 1957). Although some studies about ploidy levels of deciduous azalea species have been made, ploidy level of their cultivars is little known.

In the present study, many polyploid cultivars were identified in both evergreen and deciduous azaleas. This is the first description of tetraploids in Knap Hill Hybrid, 
triploid in inter-group hybrid between Belgian Hybrid and Hirado Hybrid and mixoploids in Satsuki Hybrid. Satsuki Hybrid 'Taihei', which had been identified to be a tetraploid by chromosome counting, also showed tetraploidy through the present flow cytometric analysis. Although the origin of these polyploids was not studied, unreduced gametes have been considered to be indispensable in the formation of polyploid azalea cultivars (Pryor and Frazier. 1970; Heursel and De Roo, 1981). On the other hand, there are some reports of tetraploid sports generating from diploid cultivars in Satsuki Hybrid and Belgian Hybrid (Hosoda et al., 1953; De Schepper et al., 2001b).

Four mixoploid cultivars in Satsuki Hybrid, of which the leaves consisted of both diploid and tetraploid cells, were identified through flow cytometric analysis with young leaves. It is known that guard cells on the epidermal layer originate from L1, pollen grains from L2 and adventitious roots from L3. Since the mixoploid 'Shinsen' had (1) slightly smaller guard cells than diploid evergreen azaleas, (2) pollen grains as large as those of tetraploid 'Hoshun' and (3) only one tetraploid (4C) peak in flow cytometric analysis of the roots, it must be diploid in the L1 and tetraploid in the L2 and L3. With the same approach, it is judged that the mixoploid 'Miharu' must include diploid cells in the L1 and/or L2 and tetraploid cells in the L1, L2 and/or L3.

Mixoploid 'Shinsen' is a sport from diploid evergreen azalea 'Gyoko'. 'Shinsen' has rounder leaves and thicker petals than 'Gyoko'. Since 'Shinsen' produced triploid 'Goka' in the cross with diploid 'Takarabune', it would produce $2 \mathrm{x}$ gamete. Although 'Koyo' and 'Miharu' are the sports from 'Suisen' and have different color patterns in their flowers, they have the same ploidy patterns as the original cultivar 'Suisen'. Considering that the tetraploid cultivars of 'Hoshuku', 'Hoshun' and 'Taikonotsuki' are the hybrids between mixoploid 'Suisen' and tetraploid 'Eiko', 'Suisen' and its sports ('Koyo' and 'Miharu') would also produce $2 \mathrm{x}$ gamete.

Ornamental plants often contain triploid and tetraploid cultivars, in general, because polyploids have favorable characters for consumers such as vigorous growth, thick stems, large flowers and firm petals. Since some triploids are more vigorous and have bigger flowers than tetraploids, triploid cultivars have been bred in various ornamental plants such as tulips, freesias and cannas (Ukai, 2003). The same may be said in triploid Satsuki Hybrid 'Meicho', which has the largest flowers among Satsuki Hybrid cultivars, and other triploid cultivars also tend to have larger flowers than tetraploids. The same observation applies to triploid 'Euratom', a remarkably vigorous Belgian Hybrid and the most widely used as a root stock for grafting in Europe (Heursel and De Roo, 1981).

Appearance of polyploids has heavily influenced on the evolution of cultivated plants. Changing ploidy levels is followed by various morphological, physiological and genetic rearrangements. Hybridization with tetraploids of Primula malacoides, which was originally a diploid with low variability, produced attractive plants with attractive characters such as more flowers, larger flowers and more seeds per flower than diploids (Horn, 2003). Flower color variability in tetraploids of $P$. malacoides was explained by dosage effects. De Schepper et al. (2001b) revealed the close relationships among somatic polyploidy, flower coloration and flower morphology in azalea. Although only few attempts have so far been made in interploid crossings among azaleas, this suggests that hybridization with polyploids plays an important role in the expansion of plant characteristics.

\section{Intrasubgeneric and interploid crossability in evergreen and deciduous azaleas}

Many diploids, triploids, tetraploids and mixoploids were identified among azalea cultivars in this study. Crossability of polyploids in interploid crosses was clarified through intrasubgeneric crossings with evergreen and deciduous azaleas. High fertility in evergreen azaleas and high cross compatibilities among them had been described in previous reports so far as diploids were concerned (Noguchi, 1932; Akabane et al., 1971; Yamaguchi et al., 1985). Yamaguchi et al. (1985) suggested that it was better to use large-flowered species for the pollen parents when the sizes of parental flowers were apparently different. Michishita et al. (2003) proved the positive relationship between male/female style length ratio (SLR) and number of seeds per capsule in interspecific crosses among evergreen azaleas. In the present study, the degree of fertility was distinctive in different ploidy levels and cross compatibility differed individually among interploid crosses. Tetraploid evergreen azaleas were fertile as seed or pollen parents, while tetraploid deciduous azaleas had low crossability as seed parents. Higher capsule set and more perfect seeds were obtained from the $2 \mathrm{x} \times 4 \mathrm{x}$ crosses than those from the $4 \mathrm{x} \times 2 \mathrm{x}$ crosses with evergreen or deciduous azaleas. There would be a room for arguing whether tetraploids with large flowers affect on fertilization in $4 \mathrm{x} \times 2 \mathrm{x}$ crosses.

Since no capsules set in the crosses with triploids evergreen azaleas as pollen parents, it is considered that triploids are male sterile or have very low pollen fertility. Crosses of the $3 \mathrm{x} \times 2 \mathrm{x}$ set capsules most frequently and produced the largest number of seeds among interploid crossings of evergreen azaleas. It may be judged from these results that triploid evergreen azaleas have relatively high female fertility as seeds parents. The reason for the high fertility is not obvious at present. In highbush blueberries, close relatives to Rhododendron, crossabilities of triploids were very low when they were used as pollen parents (Vorsa and Ballington, 1991). However, one triploid plant was relatively fertile as a female parent in the $3 \mathrm{x} \times 4 \mathrm{x}$ and the $3 \mathrm{x} \times 6 \mathrm{x}$ crosses. The female fertility has been considered to attribute to $2 \mathrm{x}$ gamete production in the triploid.

Ploidy levels of the progenies obtained in the present study remain to be proved. Since polyploids tend to form gametes with asymmetric chromosome set more frequently than diploids, the progenies obtained from interploid crosses with polyploids might have more allele 
combinations than diploid progenies from intraploid crosses with diploids. Correlation between allele dosage effects and flower colors has been observed in tetraploids of Primura malacoides (Horn, 2003). The present intrasubgeneric and interploid crosses with evergreen and deciduous azaleas must have produced various ploids or aneuploids with high heterozygosity. They might have variegated phenotypes and be useful materials to clarify the relationships between allele dosage effects and various characteristics.

\section{ACKNOWLEDGEMENT}

The authors wish to thank Mr. Masaru Akabane for his donation of the white-flowered evergreen azalea. We also thank to Associate Professor Koichiro Gyokusen in the Laboratry of Silviculture, Faculty of Agriculture, Kyushu University for cooperation in flow cytometric analysis.

\section{REFERENCES}

Akabane, M., A. Yamanaka, D. Takashima, T. Nakatsue and Y. Nakamura 1971 On the fertility of interspecific crossing and the growth of $\mathrm{F}_{1}$ seedlings in rhododendron species. Bull. Tochigi Pref. Agri. Expt. Stn., 15: 95-102 (In Japanese)

De Schepper, S., L. Leus, M. Mertens, E. Van Bockstaele and M. De Loose 2001a Flow cytometric analysis of ploidy in Rhododendron (subgenus Tsutsusi). HortScience, $\mathbf{3 6}$ : 125-127

De Schepper, S., L. Leus, M. Mertens, P. Debergh, E. Van Bockstaele and M. De Loose 2001b Somatic polyploidy and its consequences for flower coloration and flower morphology in azalea. Plant Cell Rep., 20: 583-590

Heursel, J. and R. De Roo 1981 Polyploidy in evergreen azaleas. HortScience, 16: 765-766

Horn, W. 2003 The patterns of evolution and ornamental plant breeding. Acta Hortic., 651: 19-31

Hosoda, T., A. Moriya and M. Sarashima 1953 Chromosome number of Satsuki, Rhododendron lateritum. Genetica, 26: 407-409 (Cited from: Pryor, R. L. and L. C. Frazier 1970 Triploid azaleas of the Belgian-Indian series. HortScience, 5: 114-115)
Janaki Ammal, E. 1950 Polyploidy in the genus Rhododendron. In "The Rhododendron Year Book 1950", Royal Hort. Soc., London, pp. 92-98 (Cited from: De Schepper, S., L. Leus, M. Mertens, E. Van Bockstaele, M. De Loose, P. Debergh and J. Heursel 2001a Flow cytometric analysis of ploidy in Rhododendron (subgenus Tsutsusi). HortScience, 36: 125-127)

Kehr, A. 1987 Hybridizing azaleas. In "Azalea", ed. by F. C. Galle. Timber Press, Portland, Ore. pp. 341-350

Li, H. L. 1957 Chromosome studies in the azaleas of eastern north America. Amer. J. Bot., 44: 8-14

Michishita, A., K. Ureshino, I. Miyajima, K. Sakai and Y. Ozaki 2003 Capsule set, seed productivity and germination in interspecific crosses among evergreen azaleas. J. Fac. Agr, Kyushu Univ., 47: 283-288

Noguchi, Y. 1932 Studies of the species crosses of Japanese Rhododendron. I. On the crossability between various species and the cotyledon color of $F_{1}$ seedlings. Japan. J. Bot., 6: 103-124

Pryor, R. L. and L. C. Frazier 1970 Triploid azaleas of the Belgian-Indian series. HortScience, 5: 114-115

Rouse, J. L. 1984 Pollen storage and Rhododendron breeding. In "Pollination '84", eds. by E. G. Williams and R. B. Knox, School of Botany, University of Melbourne, Australia, pp. 185-186 (Cited from: Williams, E. G., J. L. Rouse, B. F. Palser and R. B. Knox 1990 Reproductive biology of Rhododendron. Hort. Rev., 12: 1-67)

Sax, K. 1930 Chromosome stability in the genus Rhododendron. Amer. J. Bot., 17: 247-251

Ukai, Y. 2003 Chromosome mutation and inter-ploid breeding. In "Plant breeding". University of Tokyo Press, Tokyo, pp. 222-270 (In Japanese)

Vainola, A. 2000 Polyploidization and early screening of Rhododendron hybrids. Euphytica, 112: 239-244

Van Tuyl, J. M. and K. B. Lim 2003 Interspecific hybridisation and polyploidisation as tools in ornamental plant breeding. Acta Hortic., 612: 13-22

Vorsa, N. and J. R. Ballington 1991 Fertility of triploid highbush blueberry. J. Amer. Soc. Hort. Sci., 116: 336-341

Yamaguchi, S. and Y. Hirata 1986 Breeding of pivotal hybrid plant between sects. Tsutsusi and Brachycalyx azalea in the subgenus Tsutsusi. Bull. Veg. and Ornam. Crop Res. Stn. Japan, Ser. C, 9: 59-67

Yamaguchi, S., M. Kunishige and T. Tamura 1985 Interspecific compatibility in Japanese rhododendrons. Bull. Veg. and Ornam. Crop. Res. Stn. Japan, Ser. B, 8: 87-97 (In Japanese with English Summary) 